\title{
The Unsteady Flow of a Fluid of Finite Depth with an Oscillating Bottom
}

\author{
N. Pothanna ${ }^{1^{*}}$, P. Aparna ${ }^{2}$ \\ ${ }^{1,2}$ Department of Mathematics, VNR Vignana Jyothi Institute of Engineering and Technology, \\ Hyderabad, 500090, Telangana State, India. \\ pothareddy81@gmail.com
}

Keywords: Thermo-viscous fluid, thermo-stress-viscosity coefficient, prandtl number.

\begin{abstract}
In this paper, the unsteady flow of a fluid of finite depth with an oscillating bottom is examined. The flow is assumed in the absence of viscous dissipation. The governing equations of the flow are decoupled in the velocity and temperature fields. The velocity and temperature fields have been obtained analytically. The effects of various material parameters on these fields have been discussed with the help of graphical illustrations. It is noticed that the upward thrust ( $\rho f_{y}$ ) vanishes when Reiner Rivlin coefficient of viscosity $\left(\mu_{c}\right)$ is zero and the transverse force $\left(\rho f_{z}\right)$ perpendicular to the flow direction vanishes for thermo-viscosity coefficient $\left(\alpha_{8}\right)$ is zero. The external forces generated perpendicular to the flow direction is a special feature of thermo-viscous fluid when compared to the other type of fluids.
\end{abstract}

\section{Introduction}

Considerable interest has been evinced in the recent years on the study of viscous flows because of its natural occurrence and its importance in industrial geophysical and medical applications. Some practical problems involving such studies include the percolation of water through solids, the drainage of water for irrigation, the aquifier considered by the ground water hydrologists, the reserve bed used for filtering drinking water and the seepage through slurries in drains by the sanitary engineer, the flow of liquids through ion-exchange beds, cleaning of oil-spills etc. In the physical world, the investigation of the flow of thermo-viscous flows has become an important topic due to the recovery of crude oil from the pores of reservoir rocks, the extraction and filtration of oil from wells, the oil reservoir treated by the reservoir engineer, the extraction of energy from geo-thermal regions are some of the areas in which thermo-viscous flows have been noticed.

The concept of thermo-viscous fluids which reflect the interaction between thermal and mechanical responses in fluids in motion due to external influences was introduced by Koh and Eringin in 1963. For such a class of fluids, the stress-tensor ' $t$ ' and heat flux bivector ' $h$ ' are postulated as polynomial functions of the kinematic tensor, viz., the rate of deformation tensor ' $d$ ':

$d_{i j}=\left(u_{i, j}+u_{j, i}\right) / 2$

and thermal gradient bivector ' $b$ '

$b_{i j}=\epsilon_{i j k} \theta_{k}$

where $u_{i}$ is the $i^{\text {th }}$ component of velocity and $\theta$ is the temperature field.

A second order theory of thermo-viscous fluids is characterized by the pair of thermomechanical constitutive relations:

$t=\alpha_{1} I+\alpha_{3} d+\alpha_{5} d^{2}+\alpha_{6} b^{2}+\alpha_{8}(d b-b d)$

and

$h=\beta_{1} b+\beta_{3}(b d+d b)$ 
with the constitutive parameters $\alpha_{i}, \beta_{i}$ being polynomials in the invariants of $d$ and $b$ in which the coefficients depend on density $(\rho)$ and temperature $(\theta)$ only. The fluid is Stokesian when the stress tensor depends only on the rate of deformation tensor and Fourier-heat-conducting when the heat flux bivector depends only on the temperature gradient-vector, the constitutive coefficients $\alpha_{1}$ and $\alpha_{3}$ may be identified as the fluid pressure and coefficient of viscosity respectively and $\alpha_{5}$ as that of cross-viscosity.

Flow of incompressible homogeneous thermo-viscous fluids satisfies the usual conservation equations:

Equation of continuity

$v_{i, i}=0$

Equation of momentum

$\rho\left[\frac{\partial v_{i}}{\partial t}+v_{k} v_{i, k}\right]=\rho F_{k}+t_{j i, i}$

and the energy equation

$\rho c \dot{\theta}=t_{i j} d_{i j}-q_{i, i}+\rho \gamma$

where

$F_{k}=k^{\text {th }}$ Component of external force per unit mass,

$c=$ Specific heat,

$\gamma=$ Thermal energy source per unit mass

$q_{i}=i^{\text {th }}$ Component of heat flux bivector $=\in_{i j k} h_{j k} / 2$

$t_{i j}=$ The components of stress tensor

$d_{i j}=$ The components of rate of deformation tensor

The interaction between thermal and mechanical responses of fluids in motion due to external influences was primarily observed by Koh and Eringin[6] in 1963. A systematic rational approach for such class of fluids has been developed by Green and Nagdhi[2] in 1965. Kelly [5] in 1965 examined some simple shear flows of second order thermo-viscous fluids. In 1979 Nageswara Rao and Pattabhi Ramacharyulu[12] later studied some steady state problems dealing with certain flows of thermo-viscous fluids. Some more problems of thermo-viscous flows studied by Anuradha [1] in plane, cylindrical and spherical geometries in 2006. Muthuraj and Srinivas [10] studied the problem of flow of a thermo-viscous fluid through an annular tube with constriction in 2006. Srinivas et al.[19] studied the problem of Slow steady motion of a thermo-viscous fluid between two parallel plates with constant pressure and temperature gradients in 2013. Pothanna and Srinivas et al.[18] examined the problem linearization of thermo-viscous fluid in a porous slab bounded between two fixed permeable horizontal parallel plates in the absence of thermo-mechanical interaction coefficient in 2014. Pothanna etal.[17,18] examined some steady and unsteady state problems dealing with certain flows of thermo-viscous fluids between parallel plates with various assumptions .

Motsa and Animasaun[8] studied paired quasi-linearization analysis of heat transfer in unsteady mixed convection nano fluid containing both nano particles and gyrotactic microorganisms due to impulsive motion. Motsa and Animasaun[9] examined unsteady boundary layer flow over a vertical surface due to impulsive and buoyancy in the presence of thermal-diffusion and diffusion-thermo using bivariate spectral relaxation method. Koriko et al.[7]boundary layer analysis of exothermic and endothermic kind of chemical reaction in the flow of non-darcian unsteady micropolar fluid 
along an infinite vertical surface. Animasaun[4] studied dynamics of unsteady MHD convective flow with thermo phoresis of particles and variable thermo-physical properties past a vertical surface moving through binary mixture.

Keeping this in mind the relevance and growing importance of thermo-viscous fluids in geophysical fluid dynamics, chemical technology and industry; the present paper attempts to study the variations of velocity and temperature fields on the unsteady flow of thermo-viscous fluid over a flat plate with an oscillating bottom for the various material parameters.

\section{Mathematical Formulation and Solution}

Consider the Cartesian coordinate system $\mathrm{O}(\mathrm{X}, \mathrm{Y}, \mathrm{Z})$ with the origin on oscillating plate, the Xaxis is considered in the direction of the fluid flow and $\mathrm{Y}$-axis is perpendicular to the plates. Let the second order thermo-viscous incompressible fluid of finite depth $h$ bounded by the oscillating bottom $y=0$ along the $\mathrm{X}$-direction. The fluid, otherwise at rest, responds to the fluctuations of the bottom. The periods of oscillation of the fluid response and the temperature distribution are assumed to be oscillatory with the same frequency.

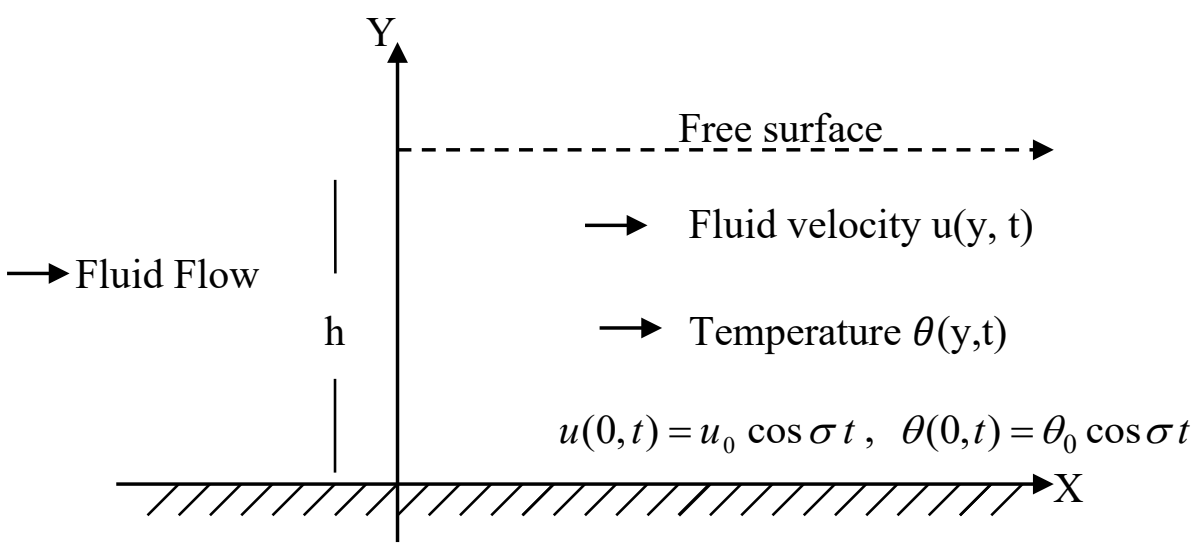

Fig. 1: Physical Model and Flow Configuration

Consider the unsteady flow be characterized by the velocity field $[u(y, t), 0,0]$ and temperature field $\theta(\mathrm{y}, \mathrm{t})$. This choice of assumption of velocity usually satisfies continuity equation. It is assumed that (i) the dissipation term $\mu\left(\frac{\partial u}{\partial y}\right)^{2}$ in the energy equation neglected (ii) the bottom is oscillating with the velocity $u_{0} \cos \sigma t$ (iii) the temperature of the bottom is oscillating with $\theta_{0} \cos \sigma t$, then the equation of momentum and energy reduces :

in the X-direction:

$$
\rho \frac{\partial u}{\partial t}=\mu \frac{\partial^{2} u}{\partial y^{2}}
$$

in the Y-direction:

$0=\mu_{c} \frac{\partial}{\partial y}\left(\frac{\partial u}{\partial y}\right)^{2}+\rho F_{y}$

in the Z- direction:

$0=\alpha_{8} \frac{\partial}{\partial y}\left(\frac{\partial \theta}{\partial y} \frac{\partial u}{\partial y}\right)+\rho F_{z}$ 
and the energy equation

$$
\rho c \frac{\partial \theta}{\partial t}=k \frac{\partial^{2} \theta}{\partial y^{2}}
$$

with the boundary conditions:

$$
\begin{aligned}
& u(0, t)=u_{0} \cos \sigma t \quad \text { and } \quad \frac{\partial u}{\partial y} /(h, t)=0 \\
& \theta(0, t)=\operatorname{Re} \theta_{0} \exp (i \sigma t) \quad \text { and } \quad \theta(h, t)=0
\end{aligned}
$$

Let the velocity distribution be assumed in the form

$$
u(y, t)=\operatorname{Re} . f(y) \exp (i \sigma t)
$$

Substituting (7) in (1) and using the boundary conditions (5), the velocity distribution is obtained as

$$
\begin{aligned}
u(y, t) & =\operatorname{Re} \cdot u_{0}\left[\frac{\cosh m(h-y)}{\cosh (m h)}\right] e^{i \sigma t} \\
& =u_{0}\left[P_{1}(y) \cos \sigma t-Q_{1}(y) \sin \sigma t\right]
\end{aligned}
$$

Where $m=\frac{\delta i \rho}{\mu}$

$$
\text { here } \begin{aligned}
P_{1}(y) & =\frac{\cos \delta(2 h-y) \cosh (\delta y)+\cos (\delta y) \cosh \delta(2 h-y)}{\cosh 2 \delta h+\cos 2 \delta h} \\
Q_{1}(y) & =\frac{\sin \delta(2 h-y) \sinh \delta y-\sin \delta y \sinh \delta(2 h-y)}{\cosh 2 \delta h+\cos 2 \delta h}
\end{aligned}
$$

The temperature distribution is assumed as $\theta(y, t)=\operatorname{Re} \cdot g(y) \exp (i \sigma t)$

From the equations (4) and (6), we obtain the temperature distribution as

$$
\begin{aligned}
\theta(y, t) & =\operatorname{Re} \cdot \theta_{0} \frac{\sinh \left(p_{r} m^{2}(h-y)\right)}{\sinh \left(p_{r} m^{2} h\right)} \\
& =\theta_{0}\left[P_{2}(y) \cos \sigma t-Q_{2}(y) \sin \sigma t\right]
\end{aligned}
$$

with $p_{r}=\frac{\mu c}{k}$ is the prandtl number.

$$
\begin{aligned}
& P_{2}(y)=\frac{\cos \left(\delta \sqrt{p_{r}} y\right) \cosh \left(\delta \sqrt{p_{r}}(2 h-y)\right)-\cosh \left(\delta \sqrt{p_{r}} y\right) \cos \left(\delta \sqrt{p_{r}}(2 h-y)\right)}{\cosh 2 \delta \sqrt{p_{r}} h-\cos 2 \delta \sqrt{p_{r}} h} \\
& Q_{2}(y)=\frac{\sinh \left(\delta \sqrt{p_{r}} y\right) \sin \left(\delta \sqrt{p_{r}}(2 h-y)\right)-\sin \left(\delta \sqrt{p_{r}} y\right) \sinh \left(\delta \sqrt{p_{r}}(2 h-y)\right)}{\cosh 2 \delta \sqrt{p_{r}} h-\cos 2 \delta \sqrt{p_{r}} h}
\end{aligned}
$$

The normal thrust generated on the plate $y=0$, due to the cross-viscosity $\left(\mu_{c}\right)$ is

$$
\rho f_{y} /(y=0)=2 \mu_{c}(\delta)^{3} h^{2} \frac{\cos 2 \delta h-\cosh 2 \delta h}{(\cos 2 \delta h+\cosh 2 \delta h)^{2}}\left[\begin{array}{l}
(\sinh 2 \delta h-\sin 2 \delta h)(1-\cos 2 \sigma t)- \\
(\sinh 2 \delta h+\sin 2 \delta h) \sin 2 \sigma)
\end{array}\right]
$$


and the transverse force generated perpendicular to plate fluctuations is

$$
\rho f_{z} /(y=0)=2 \mu_{0} \alpha_{8} \mu_{c}(\delta)^{3} \sqrt{p_{r}}\left\{\begin{array}{l}
\frac{\cos 2 \delta h-\cosh 2 \delta h}{(\cos 2 \delta h+\cosh 2 \delta h)\left(\cos 2 \delta h \sqrt{p_{r}}-\cosh 2 \delta h \sqrt{p_{r}}\right)} \\
{\left[\begin{array}{l}
\left(\sin 2 \delta h \sqrt{p_{r}}-\sinh 2 \delta h \sqrt{p_{r}}\right) \sin ^{2} \sigma- \\
\left(\sin 2 \delta h \sqrt{p_{r}}+\sinh 2 \delta h \sqrt{p_{r}}\right) \sin \sigma t \cos \sigma t
\end{array}\right]+} \\
\sqrt{p_{r}} \frac{\sin 2 \delta h-\sinh 2 \delta h}{\cos 2 \delta h+\cosh 2 \delta h} \sin \sigma t(\sin \sigma t-\cos \sigma t)-
\end{array}\right\}
$$

\section{Results and Discussions}

The Graphs shown below are generated using MATLAB Code for the fixed values $\mu=1, v=1$, $\delta=1$ and $c=1$. Fig. 2 and Fig. 3 shows the variations of velocity profiles with the different values of time parameter $(\mathrm{t})$. It can be noted that the fluid velocity is fluctuating with the period $\left(=\frac{2 \pi}{\sigma}\right)$. In Fig.2, it is observed that the velocity profile variations are oscillating with the frequency $\sigma=1$ (i.e. the velocity of the fluid is oscillating with the period $2 \pi$ ). It is also noticed from the Fig. 3 that the velocity profile variations are oscillating with the frequency $\sigma=2$ (i.e. the velocity is oscillating with the period $\pi$ ).

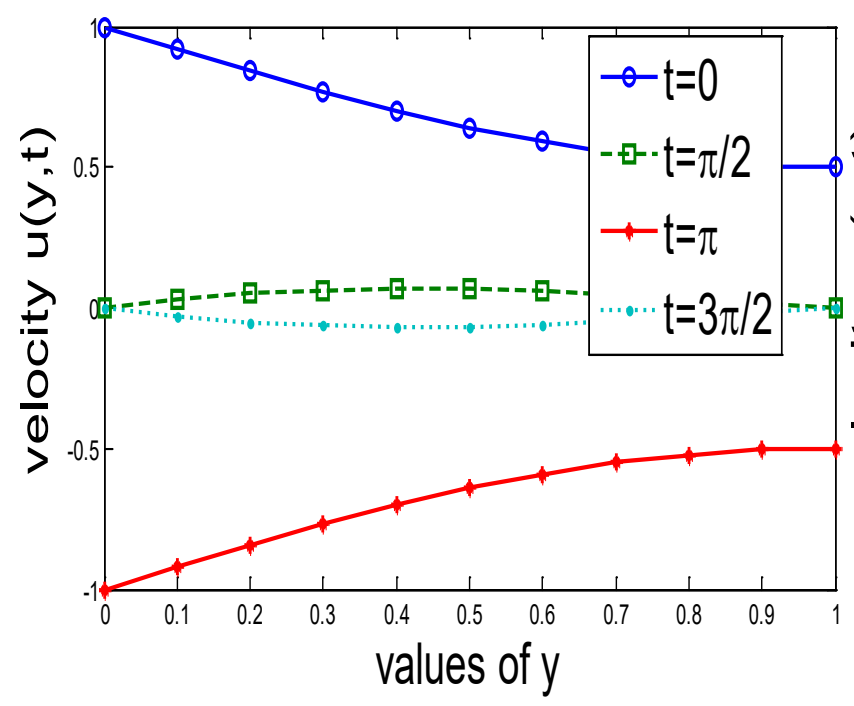

Fig. 2: Velocity versus $t, \sigma=1$

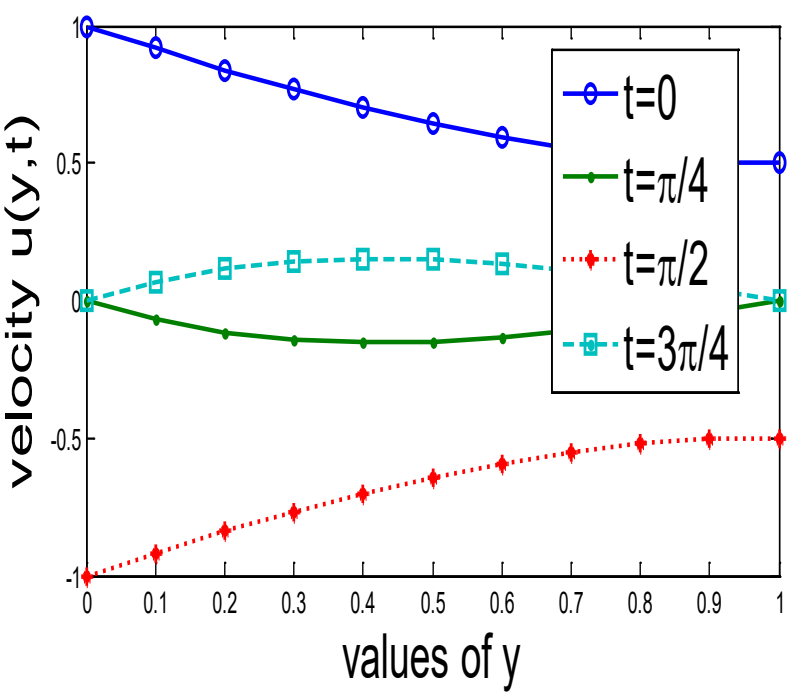

Fig. 3: Velocity versus $t, \sigma=2$

The temperature distribution variations with different values of time parameter $(\mathrm{t})$ and prandtl number $\left(p_{r}\right)$ are illustrated in Fig.5, Fig.6 and Fig.7. It shows that the temperature distributions moving towards centre for the values increases from $t=0$ to $t=\frac{\pi}{2}$ and the temperature distributions shifting towards centre from down for the values increases from $t=\pi$ to $t=\frac{3 \pi}{2}$. The temperature distribution variations with the various values of prandtl number are shown in Fig.7. This shows that the temperature of the fluid decreases as the values of prandtl number $\left(p_{r}\right)$ increases from 1 to 3 . 


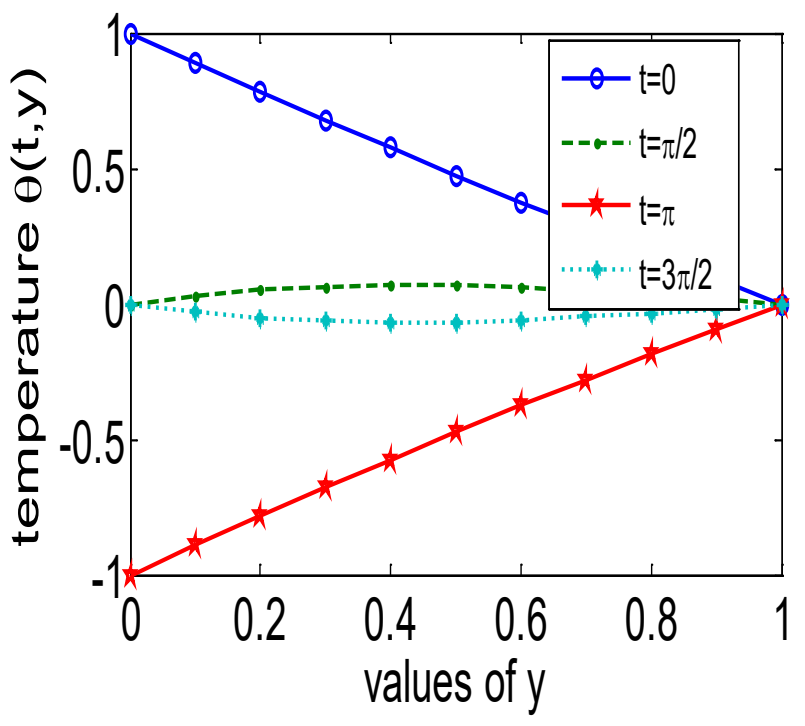

Fig. 4: Temperature versus $t, p_{r}=1, \sigma=1$

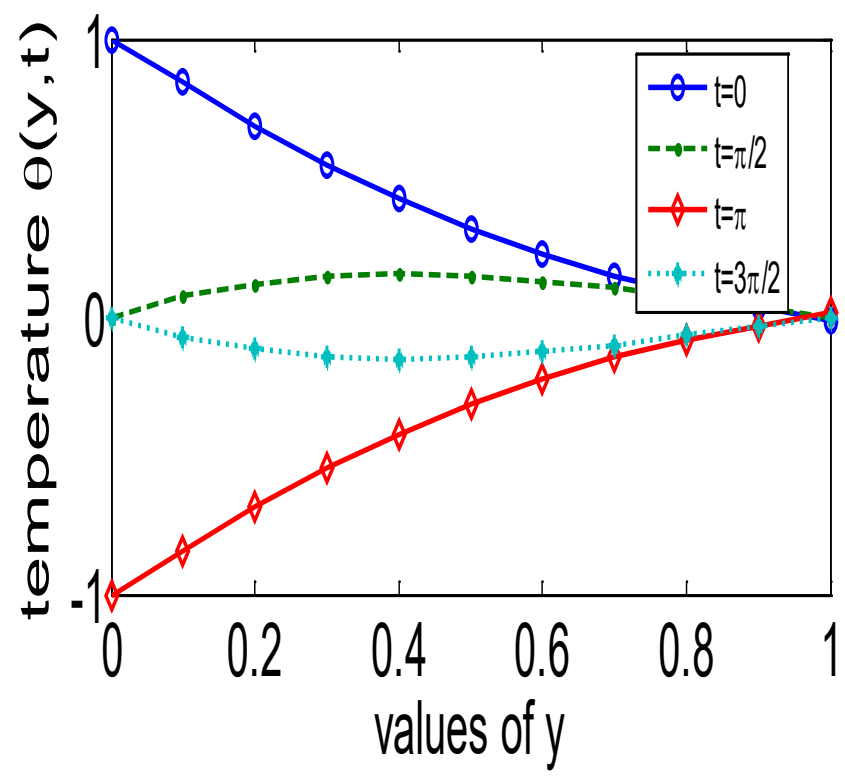

Fig. 6: Temperature versus $t, p_{r}=3, \sigma=1$

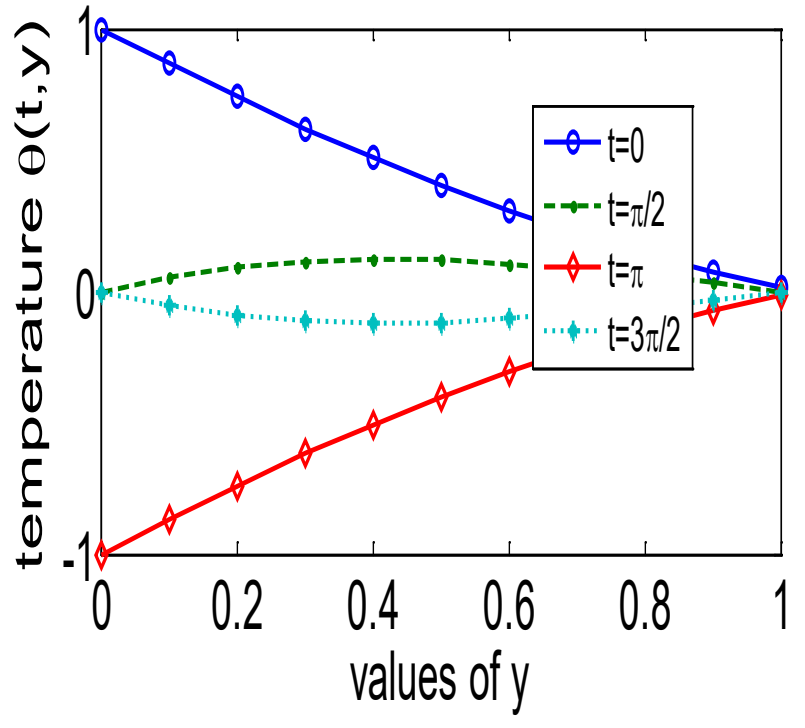

Fig. 5: Temperature versus $t, p_{r}=2, \sigma=1$

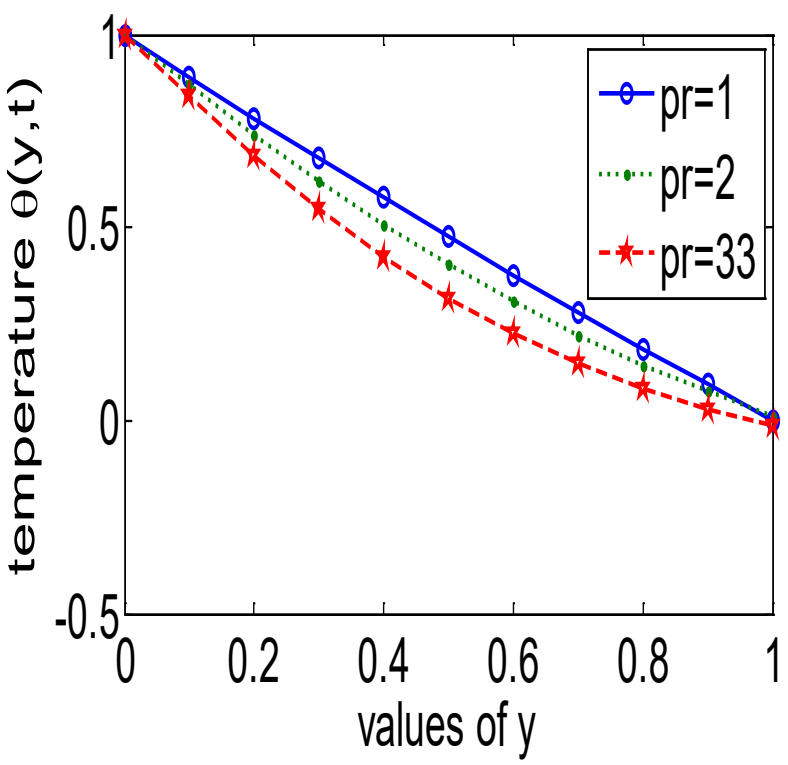

Fig. 7: Temperature versus $p_{r}, t=0, \sigma=1$

\section{Conclusion}

The present investigation deals with an unsteady flow of a thermo-viscous incompressible fluid of finite depth with an oscillating bottom. The following conclusions are drawn from the present study.

- It is noticed that the upward thrust $\left(\rho f_{y}\right)$ vanishes for Reiner Rivlin coefficient of viscosity $\left(\mu_{c}\right)$ is zero and the transverse force $\left(\rho f_{z}\right)$ perpendicular to the flow direction is vanishes for thermo-viscosity coefficient $\left(\alpha_{8}\right)$ is zero.

- Temperature of the fluid decreases as increases of prandtl number $\left(p_{r}\right)$.

- Temperature of the fluid increases as increases of time parameter $(t)$. 


\section{References}

[1] K. Anuradha, "On steady and unsteady flows of thermo-viscous Fluids", Ph. D thesis , J.N.T.U. Hyderabad, 2006.

[2] A.E.Green and P.M. Naghdi, "A dynamical theory of interacting Continua" , Int. J. Engg. Sci., vol.3, pp.231-241, 1965.

[3] A.E.Green and P.M. Naghdi, "A new thermo-viscous theory for fluids", Journal of NonNewtonian Fluid Mechanics, vol. 56, No.3, pp.289-306, March 1995.

[4] I. L. Animasaun, "Dynamics of Unsteady MHD Convective Flow with Thermophoresis of Particles and Variable Thermo-Physical Properties past a Vertical Surface Moving through Binary Mixture”, Open Journal of Fluid Dynamics, vol.5, pp.106-120, 2015.

[5] P.D.Kelly, "Some viscometric flows of incompressible thermo-viscous fluids", Int. J. Engg. sci., vol.2, pp.519-537, 1965.

[6] S.L.Koh and A. C. Eringin, "On the foundations of non-linear thermo-elastic fluids", Int. J. Engg. Sci., vol.1, pp.199 -229, 1963.

[7] O.K. Koriko, A.J. Omowaye, I.L. Animasaun, I.O. Babatunde, "Boundary Layer Analysis of Exothermic and Endothermic Kind of Chemical Reaction in the Flow of Non-Darcian Unsteady Micropolar Fluid Along an Infinite Vertical Surface", International Journal of Engineering Research in Africa, vol.28, pp.90 - 101, 2017.

[8] S. S. Motsa, I. L. Animasaun, "Paired Quasi-Linearization Analysis of Heat Transfer in Unsteady Mixed Convection Nano fluid Containing Both Nanoparticles and Gyrotactic Microorganisms Due to Impulsive Motion", Journal of Heat Transfer, vol.138(11), pp.114503-114503-8, 2016.

[9] S. S. Motsa, I. L. Animasaun, "Unsteady Boundary Layer Flow over a Vertical Surface due to Impulsive and Buoyancy in the Presence of Thermal-Diffusion and Diffusion-Thermo using Bivariate Spectral Relaxation Method", Journal of Applied Fluid Mechanics vol.9(5), pp.2605-2619, 2016.

[10] R. Muthuraj and S. Srinivas, "Flow of a Thermo-viscous Fluid through an Annular Tube with Constriction”, Defence Science Journal, vol. 57, No. 5, pp. 653-659, September 2007.

[11] E. Nagaratnam, "Some steady and unsteady flows of thermo- viscous fluids", $\mathrm{Ph} . \mathrm{D}$ thesis, J.N.T.U Hyderabad, 2006.

[12] P.Nageswara Rao, "Some problems in thermo-viscous fluid Dynamics", Ph. D thesis, K.U. Warangal, 1979.

[13] P.Nageswara rao and N.Ch. Pattabhi Ramacharyulu, "Steady flow of a second order thermoviscous fluid over an infinite plate", Proc.Ind.Acad.Sci., vol.88A, Part III No.2, pp .157-162, 1979.

[14] P.Nageswara rao and N.Ch. Pattabhi Ramacharyulu, "Steady flow of a thermo-viscous fluid through straight tubes", Journal of Ind. Inst. Sci. , vol. 61(B), pp.89-102, June 1979.

[15] P.Nageswara rao and N.Ch. Pattabhi Ramacharyulu, "A note on steady slow motion of thermo-viscous fluid through a circular tube", Indian journal of pure and appl. Math., vol.11(4), pp.487-491, April 1980.

[16] N.Ch. Pattabhi Ramacharyulu, "Problems in the steady of non-Newtonian Fluid Dynamics", Ph. D thesis, O. U. Hyderabad, 1966. 
[17] N. Pothanna, P.Nageswara rao and N.Ch. Pattabhi Ramacharyulu, "Flow of slightly thermoviscous fluid in a porous slab bounded between two permeable parallel plates", Int. J. Adv. Appl. Math. and Mech., vol.2(3), pp. 1-9, 2015.

[18] N. Pothanna, J. Srinivas, P.Nageswara rao and N.Ch. Pattabhi Ramacharyulu, "Linearization of thermo-viscous fluid in a porous slab bounded between two fixed permeable horizontal parallel plates in the absence of thermo-mechanical interaction coefficient", International Journal of Modern Trends in Engineering and Research, vol.1(5). pp. 412-424, 2014.

[19] J. Srinivas, N. Pothanna, P.Nageswara rao and N.Ch. Pattabhi Ramacharyulu, "Slow steady motion of a thermo-viscous fluid between two parallel plates with constant pressure and temperature gradients", International Journal of Research in Engineering and Technology, vol.2(11), pp.294-299, 2013. 